\title{
FAUNA DE VERTEBRADOS EOCRETÁCICOS DE UM AFLORAMENTO DA BACIA DE LIMA CAMPOS, CEARÁ, BRASIL
}

\author{
FELIPE LIMA PINHEIRO, ANA EMILIA QUEZADO DE FIGUEIREDO, DANIELCOSTAFORTIER \\ Departamento de Paleontologia e Estratigrafia, IGeo, UFRGS, Cx.P. 15001, 91501-970, Porto Alegre, RS, Brasil. \\ fl_pinheiro@yahoo.com.br,aquezado@yahoo.com.br,danielcfortier@yahoo.com.br
}

MARIA SOMÁLIA SALES VIANA

Curso de Biologia, Universidade Estadual Vale do Acaraú, Sobral, CE, Brasil.somalia_viana@hotmail.com

\author{
CESAR LEANDRO SCHULTZ \\ Departamento de Paleontologia e Estratigrafia, IGeo, UFRGS, Cx.P. 15001, 91501-970, Porto Alegre, RS, Brasil. \\ cesar.schultz@ufrgs.br
}

\begin{abstract}
THE EARLY CRETACEOUS VERTEBRATE FAUNA FROM AN OUTCROP OF THE LIMA CAMPOS BASIN, CEARÁ, BRAZIL. New vertebrate finds, probably Neocomian (Early Cretaceous) in age, from an outcrop near the locality of Cascudo, Municipality of Icó, Ceará State, are added to the abundant and well-known remains of ganoid scales attributed to Lepidotes sp. in the Lima Campos Basin. These new specimens comprise remains of coelacanth fishes (including a right fragmented mandibular ramus and fragmented remains of a quadrate, an angular and a prootic), three hybodont teeth identified as Planohybodus sp., and a complete crocodilomorph osteoderm. These new finds were discovered in the fine-grained red sandstone from Malhada Vermelha Formation. This study reveals the high and still little explored paleontological richness of the Lima Campos Basin, and points to the fossiliferous potential of others small northeastern Brazilian inner basins.
\end{abstract}

Key words: Malhada Vermelha Formation, Lima Campos Basin, Hybodontidae, Semionotidae, Mawsoniidae, Crocodylia.

\begin{abstract}
RESUMO - Novos achados de vertebrados, provavelmente de idade Neocomiana (Eocretáceo), provenientes de um afloramento da região de Cascudo, Município de Icó, Ceará, somam-se aos já conhecidos e abundantes registros de escamas ganoides, relacionadas a peixes do gênero Lepidotes sp., para a bacia de Lima Campos. As novas descobertas compreendem registros de peixes celacantiformes (incluindo um ramo mandibular direito fragmentado, além de ossos isolados como quadrado, angular e proótico), três dentes de tubarões hibodontídeos do gênero Planohybodus sp., e um osteodermo de crocodilomorfo. Todos os novos materiais foram coletados em um arenito fino avermelhado, de origem fluvial, pertencente à Formação Malhada Vermelha. Este estudo revela a elevada e ainda pouca explorada riqueza paleontológica da bacia de Lima Campos, e assinala para o potencial fossilífero das demais pequenas bacias interiores do nordeste brasileiro.
\end{abstract}

Palavras-chave: Formação Malhada Vermelha, bacia de Lima Campos, Hybodontidae, Semionotidae, Mawsoniidae, Crocodylia.

\section{INTRODUÇÃO}

O Estado do Ceará, historicamente, vem sendo alvo de intensas pesquisas paleontológicas, o que se deve, principalmente, à riqueza fossilífera da bacia do Araripe, inigualável depósito de fósseis do período Cretáceo, reconhecido globalmente tanto pela quantidade quanto pela qualidade de seu material fossilífero (Maisey, 1991).

A excepcionalidade da bacia do Araripe, entretanto, contribuiu para que outras pequenas bacias sedimentares da região centro-leste do Estado do Ceará (Iguatu, Lima Campos, Icó e Malhada Vermelha), fossem alvo de pesquisas mais esporádicas, embora estas preservem, em suas rochas, um importante e ainda pouco explorado registro de vida cretácica.

Estas pequenas bacias fazem parte do conjunto de bacias interiores do nordeste do Brasil, cuja gênese está associada aos processos de rifteamento entre as placas Sul-Americana e Africana. Tais processos ocorreram durante o final do período Jurássico e início do Cretáceo, propiciando a instalação do Oceano Atlântico Sul. Os sedimentos que as compõem são provavelmente de idade Neocomiana (Eocretáceo), depositados em leques aluviais, sistemas fluviais entrelaçados e lacustres.

Os escassos trabalhos científicos que tratam da geologia e paleontologia dessas bacias, iniciados com as obras de 
Small (1913) e Moraes (1924), já registraram a presença de pólens, esporos, fragmentos vegetais, icnofósseis de invertebrados e vertebrados, conchostráceos, ostracodes, moluscos, possíveis larvas de insetos, fragmentos de peixes e crocodilomorfos (Tinoco \& Mabesoone, 1975; Vogel, 1976a,b; Mussa et al., 1991; Cavalcanti \& Viana,1992; Leonardi \& Spezzamonte, 1994; Fortier \& Schultz, 2006).

No final de 2005, uma expedição geológica executada por pesquisadores da Universidade Federal do Rio Grande do Sul (UFRGS) realizou estudos de cunho estratigráfico nestas pequenas bacias interiores - que resultaram no trabalho de Arima (2007) - registrando a presença de diversos afloramentos com ocorrência de fósseis, alguns dos quais foram descritos por Fortier (2008) e Fortier \& Schultz (2009). Em 2007, 2008 e 2009 foram realizadas três novas expedições à região, visando prospecção e coleta de fósseis. As duas primeiras envolveram pesquisadores da UFRGS e Universidade Estadual do Ceará (UECE) e, a terceira, fez parte de um projeto científico conjunto entre a UFRGS e a Universidade Estadual Vale do Acaraú (UVA). Estas expedições propiciaram a identificação daqueles afloramentos mais interessantes, do ponto de vista paleontológico, nos quais foi então intensificada a coleta de espécimes fósseis. Como resultado, além da ampliação da quantidade de material prospectado, inclusive com a identificação de novos táxons, foram também reunidos dados de interesse tafonômico (Figueiredo, 2009).

O presente trabalho enfoca os achados provenientes de um destes afloramentos mais fossilíferos da bacia de Lima Campos, localizado na região de Cascudo, Município de Icó, Ceará, constituído por arenito fino avermelhado, de origem fluvial, pertencente à Formação Malhada Vermelha.

Abreviações institucionais. UECE, Universidade Estadual do Ceará; UFC, Universidade Federal do Ceará; UFRGS, Universidade Federal do Rio Grande do Sul.

\section{LOCALIZAÇÃO DO AFLORAMENTO E CONTEXTO GEOLÓGICO}

O local das coletas tem sido informalmente designado de "afloramento Cascudo", em função de sua proximidade da localidade de Cascudo, Município de Icó, Ceará. Localiza-se às margens da rodovia CE-282, que liga as cidades de Icó e Iguatu, com coordenadas UTM 24M-492215E/9291072N (Figura 1).

A estrada corta o afloramento, que possui cerca de $60 \mathrm{~m}$ de comprimento e 4,5 m de espessura, com orientação NWSE. O afloramento, pertencente à Formação Malhada Vermelha, é constituído, na base, por intercalações de siltitos avermelhados maciços ou laminados com níveis centimétricos de arenito fino com laminação cruzada cavalgante (climbing ripples) e, no topo, por arenitos médios com estratificação cruzada acanalada (Figura 2). Estes níveis estão recobertos por sedimentos terciários da Formação Moura. Estas litologias, típicas da Formação Malhada Vermelha, constituem uma associação de fácies de canal, espraiamento de crevasse e planície de inundação (Arima, 2007; Figueiredo,
2009), que representam uma sucessão sedimentar cíclica acompanhando mudanças climáticas. Os fósseis encontram-se nas camadas de arenito fino e, na porção superior, também nos arenitos médios.

\section{CONTEÚDO FOSSILÍFERO}

Foram observados, no afloramento Cascudo, três níveis fossilíferos em arenitos finos intercalados com siltitos, além de um quarto nível no arenito médio superior (Figura 2). São abundantes as superfícies com icnofósseis de invertebrados e escamas de peixes. Um dos níveis de arenito fino apresenta a maior variedade de formas, incluindo dentes de tubarões, ossos cranianos de peixes celacantiformes e semionotiformes, além de um osteodermo de crocodilomorfo. No arenito médio do topo da sequência foram encontrados fragmentos ósseos atribuídos a celacantiformes, além de escamas rômbicas de peixes.

A análise do material coletado, levando-se em consideração o grau de preservação, a disposição dos espécimes nas diferentes fácies e os táxons que foram encontrados associados, permitiu algumas inferências tafonômicas e paleoecológicas. Os níveis fossilíferos estão associados aos arenitos, sendo que os fósseis encontram-se dispersos na matriz, desarticulados e isolados. Os elementos fragmentados não mostram arredondamentos em suas extremidades, indicando que os bioclastos foram transportados apenas por curtas distâncias. Reforçando esta hipótese, foram preservadas, por exemplo, as estruturas de ornamentação de ossos cranianos de celacantiformes e as estrias e o esmalte nos dentes de tubarões. Muitas das escamas apresentam a camada externa de ganoína fraturada, indicando um possível ressecamento post mortem. No entanto, não é descartada a possibilidade de o fraturamento ter se dado durante o processo de transporte ou mesmo após a diagênese. Com base nessa evidência e na presença de elementos ósseos fraturados e desarticulados, entende-se que os restos de vertebrados aqui descritos devem ter ficado expostos por algum tempo, na superfície, antes de sua acumulação e soterramento definitivos (Figueiredo, 2009). Entretanto, não foi observada nenhuma evidência de exposição subaérea prolongada (como gretas de ressecamento ou marcas de raízes) nos sedimentos finos de planície de inundação.

Uma integração preliminar dos dados sedimentares e tafonômicos sugere pelo menos dois eventos tafonômicos recorrentes antes da acumulação e do soterramento definitivos: (i) acumulação dos ossos de vertebrados (elementos desarticulados e ganoína fraturada) que talvez tenham sido expostos em algum momento anterior ao soterramento final; e (ii) produção dos icnofósseis de invertebrados (tempo de menor taxa de sedimentação e de oxigenação do fundo).

Paleoecologicamente, os táxons de vertebrados encontrados constituem-se numa fauna aquática formada por peixes ósseos e cartilaginosos com a presença de crocodilomorfos (Figueiredo et al., 2008), sugerindo que este afloramento representa um registro de depósitos continentais de água doce. 


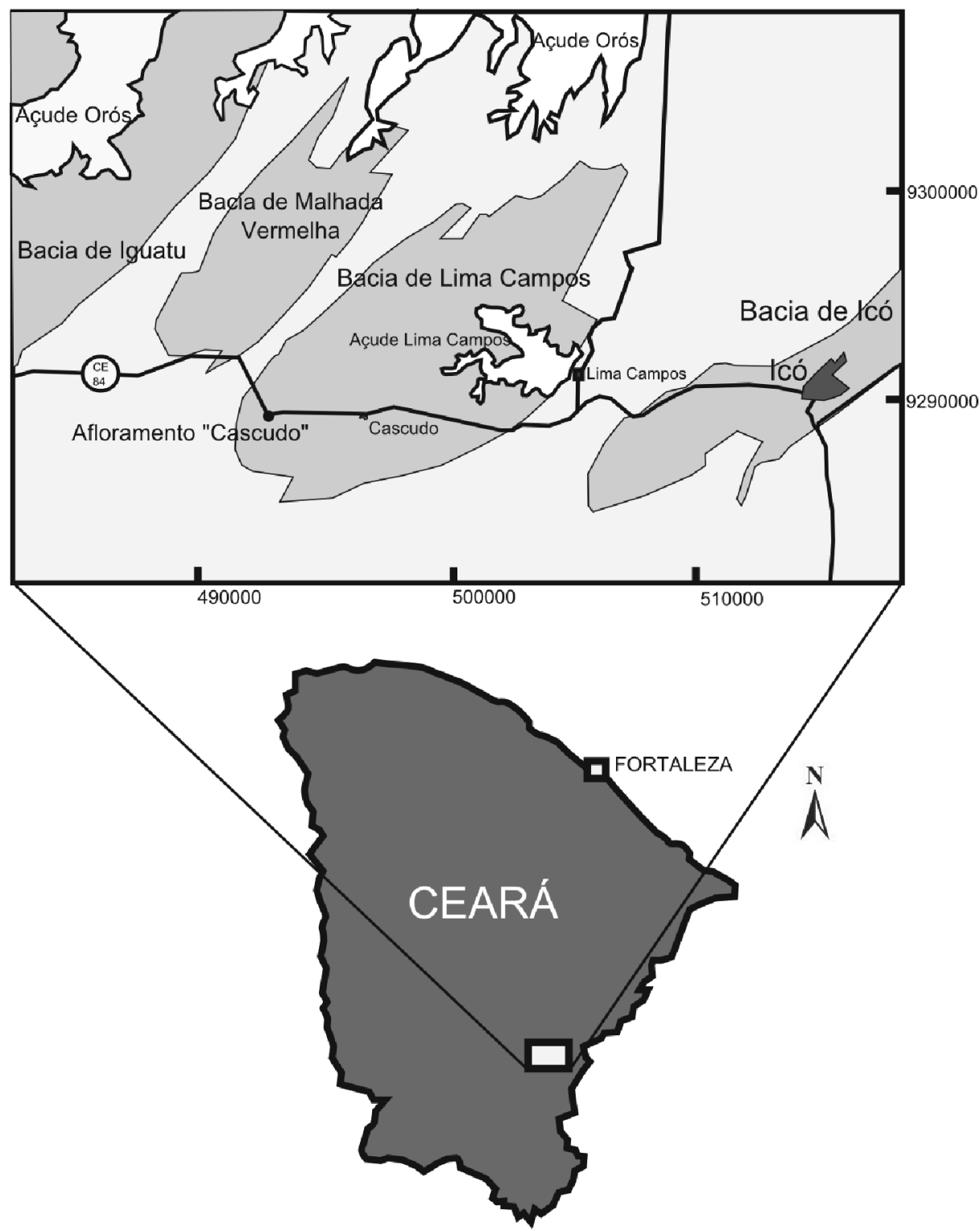

Figura 1. Localização do afloramento "Cascudo", bacia de Lima Campos, Ceará.

Figure 1. Location map of the "Cascudo" outcrop, Lima Campos Basin, Ceará State.

\section{SISTEMÁTICA PALEONTOLÓGICA}

EUSELACHII Hay, 1902

HYBODONTOIDEA Owen, 1846 sensu Zangerl, 1981 HYBODONTIDAE Owen, 1846

Planohybodus Rees \& Underwood, 2008

Planohybodus sp.

(Figuras 3A-F)

Material referido. Três dentes incompletos, UFRGSPV0021K, 22K; UECE-LP0002LC.

Descrição. Dentes de pequeno tamanho, tendo o menor um pouco mais de $4 \mathrm{~mm}$ e o maior $9 \mathrm{~mm}$ de altura. As coroas são elevadas, achatadas lábio-lingualmente e largas, com bordas cortantes bem desenvolvidas e ausência de serrilhas. Um dos exemplares (UFRGS-PV0021K) possui a cúspide central completa, enquanto que, nos outros dois, falta a extremidade. Os dentes são pronunciadamente estriados, sendo que, em um dos exemplares (UECE-LP0002LC), as estrias restringem-se à base, não havendo pronunciamento expressivo destas em direção à extremidade da cúspide central. Já nos outros dois espécimes (UFRGS-PV0021K, 22K), as estrias são mais desenvolvidas lingualmente, chegando quase ao topo da cúspide central, enquanto que, labialmente, atingem pouco mais da metade da cúspide central. Em todos os exemplares há uma inclinação da cúspide central em direção à face lingual (Figuras 3C,F). Todos os dentes estão fraturados em suas bases, impedindo uma descrição detalhada dessa região. Os dentes aqui descritos são referidos ao gênero Planohybodus sp. por apresentarem cúspides centrais altas, largas e planas, bordas cortantes bem desenvolvidas e fraca ornamentação. 


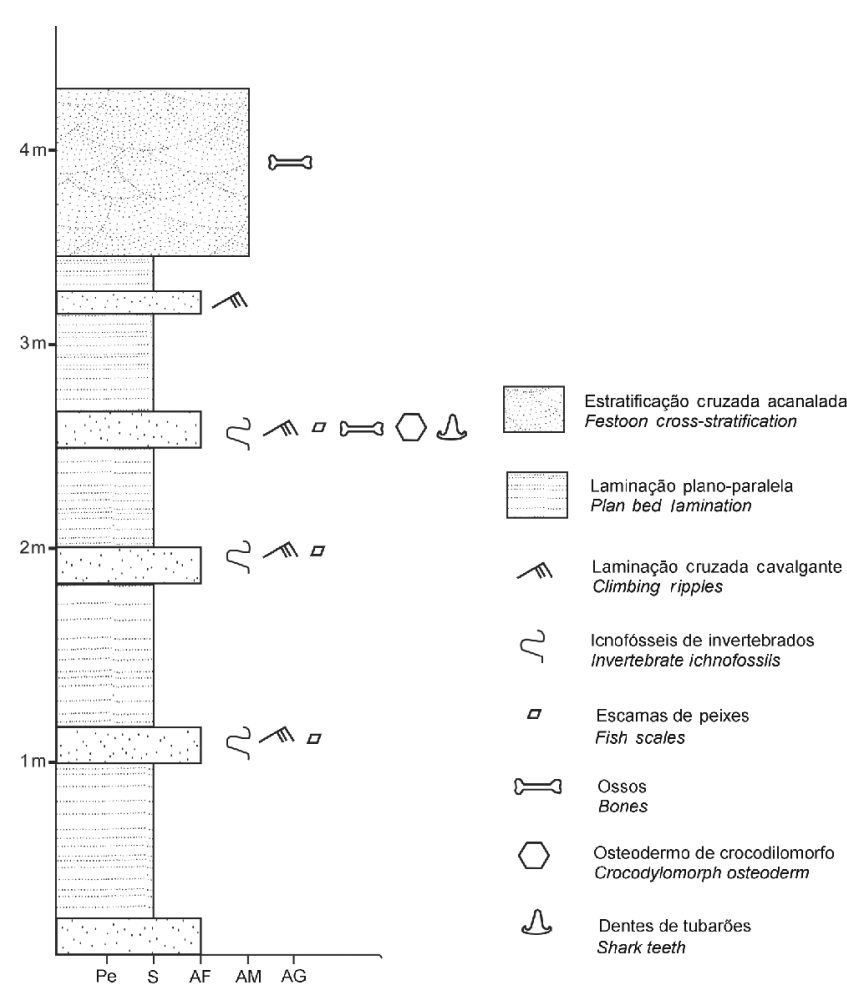

Figura 2. Perfil estratigráfico do afloramento "Cascudo". Abreviaturas: $\mathbf{P e}$, pelito; $\mathbf{S}$, siltito; $\mathbf{A F}$, arenito fino; $\mathbf{A M}$, arenito médio; AG, arenito grosso.

Figure 2. Stratigraphic section of the "Cascudo" outcrop. Abbreviations: Pe, pelite; S, siltite; AF, fine sandstone; AM medium sandstone; AG, coarse sandstone.

Discussão. O gênero Planohybodus foi proposto por Rees \& Underwood (2008), no qual foi incluída a nova espécie $P$. peterboroughensis Rees \& Underwood, 2008 e, também, $P$. grossiconus (Agassiz, 1843), hibodontídeo anteriormente referido a Hybodus Agassiz, 1837.

As características encontradas nas cúspides afastam os novos achados de outros tubarões hibodontídeos, como Hybodus e Egertonodus Maisey, 1987, pois estes últimos apresentam a cúspide central delgada, com secção transversal quase circular, aspectos morfológicos que unem Hybodus e Egertonodus na subfamília Hybodontinae (Rees, 2008). Outros gêneros filogeneticamente mais próximos de Planohybodus, como Secarodus Rees \& Underwood, 2008 e Priohybodus d'Erasmo, 1960, também podem ser descartados, já que apresentam cúspides laterais fortemente divergentes e, geralmente, possuem borda cortante serrilhada. Apenas uma espécie de Planohybodus, P. ensis (Woodward, 1916), é documentada para o Eocretáceo (BerrisianoBarremiano) da Inglaterra. Entretanto, os dentes aqui descritos diferem de $P$. ensis por apresentarem ornamentação mais proeminente e bordas cortantes sem serrilhas.

Este novo achado tem importantes implicações bioestratigráficas, biogeográficas e paleoecológicas, pois as espécies de Planohybodus supramencionadas pertencem ao Mesojurássico e Eocretáceo da Inglaterra, sendo, o material aqui descrito, o primeiro registro do gênero em outra parte do mundo. Além disso, o horizonte estratigráfico onde foi coletado o holótipo de Planohybodus peterboroughensis não é conhecido, enquanto o material aqui apresentado é proveniente de um sistema de água doce, fluvial. Nesse contexto, é provável que $P$. peterboroughensis também habitasse um ecossistema fluvial ou lacustre, ambos de ocorrência comum nas camadas do Bathoniano inglês (Rees \& Underwood, 2008).

Sabe-se que, entre o Triássico e o Eojurássico, os hibodontídeos constituíam o grupo dominante de seláquios, tanto em ambientes marinhos quanto continentais (Capetta et al., 1993; Carvalho, 2002). A partir do Neojurássico, no entanto, os hibodontídeos passaram a componentes menores da fauna marinha, continuando diversificados apenas em restritos ambientes marinhos e fluviais (Rees \& Underwood, 2008). Assim, com o declínio da distribuição dos hibodontídeos durante o Mesojurássico, tais formas teriam se concentrado em ambientes fluviais e lacustres em algumas partes do planeta, incluindo os sistemas fluviais do nordeste brasileiro, durante o Eocretáceo.

\section{ACTINOPTERYGII Cope, 1887 \\ NEOPTERYGII Regan, 1923 \\ SEMIONOTIFORMES Arambourg \& Bertin, 1958 \\ SEMIONOTIDAE sensu Wenz, 1999 \\ Lepidotes Agassiz, 1832 \\ Lepidotes sp.}

(Figura 4)

Material referido. UFC-LP085D, várias escamas ganoides.

Descrição. Escamas de formatos e espessuras variadas, pertencentes a partes distintas do corpo do animal. As formas variam de quase quadradas a losangulares. Apresentam uma espessa camada de ganoína e ornamentações diversas.

Discussão. A ocorrência de escamas de semionotídeos já havia sido bem documentada para as bacias de Iguatu (Vogel, 1976b) e Icó (Fortier, 2008). A espécie Lepidotes iguatuensis Vogel, 1976 é, hoje, considerada nomen nudum, por não ter sido formalmente descrita (Gallo \& Brito, 2004).

Outros registros de semionotídeos são tratados, em alguns dos trabalhos sobre as chamadas "bacias do Iguatu", como "escamas de peixe", não sendo propostas identificações taxonômicas mais específicas (Campos et al., 1979). Alguns trabalhos, como o de Beurlen \& Mabesoone (1969), Mabesoone \& Campanha (1974) e Cavalcanti \& Viana (1992) identificaram as escamas como sendo "do tipo Lepidotes".

As escamas são o elemento fóssil mais abundante no afloramento Cascudo, sendo encontradas em grande quantidade nas placas de arenito coletadas em ambos os lados do afloramento. A presença maciça de escamas e a ausência de outros materiais esqueletais (ossos, dentes) de Lepidotes parecem indicar um processo tafonômico, provavelmente causado por transporte diferencial das distintas partes do esqueleto pela corrente fluvial (seleção hidráulica).

A ocorrência de peixes do gênero Lepidotes e formas afins no Brasil é registrada para as "bacias do Iguatu", Parnaíba, Recôncavo, Almada, Sergipe-Alagoas, Araripe e 


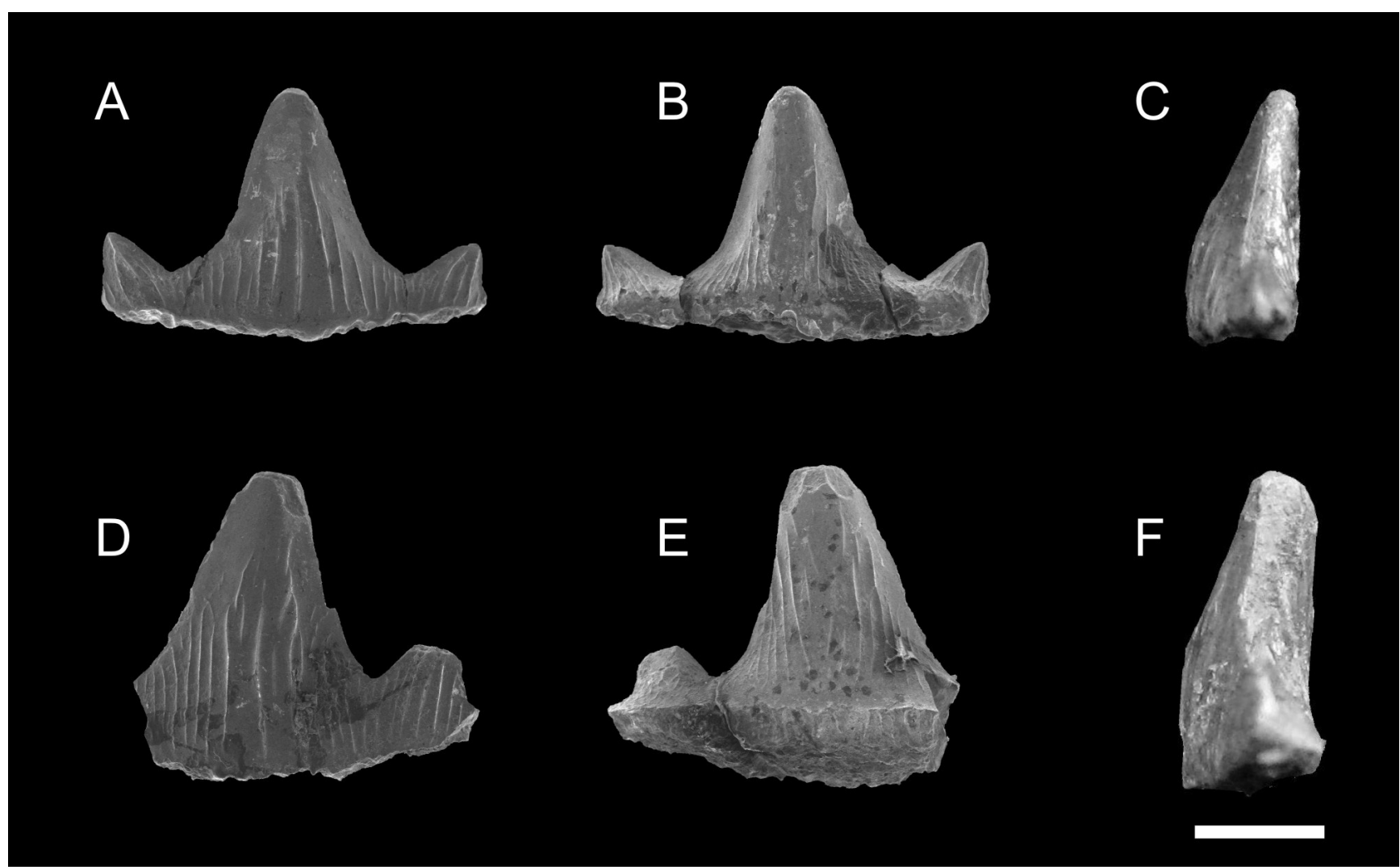

Figura 3. Dentes de Planohybodus sp. A-C, UFRGS-PV0021K: A, vista labial; B, vista lingual; C, vista lateral. D-F, UFRGS-PV0022K: D, vista labial; $\mathbf{E}$, vista lingual; $\mathbf{F}$, vista lateral. Escala $=2 \mathrm{~mm}$.

Figure 3. Teeth of Planohybodus sp. A-C, UFRGS-PV0021K: A, labial view; B, lingual view; C, lateral view. D-F, UFRGS-PV0022K: D, labial view; E, lingual view; $\mathbf{F}$, lateral view. Scale bar $=2 \mathrm{~mm}$.

Potiguar (Fortier, 2008), sendo interessante notar que todas estas bacias estão localizadas no nordeste brasileiro.

\section{OSTEICHTHYES Huxley, 1880 \\ SARCOPTERYGII Romer, 1955 \\ ACTINISTIA Cope, 1871}

Mawsoniidae Schultze, 1993

Mawsonia Woodward in Mawson \& Woodward, 1907 cf. Mawsonia sp.

(Figuras 5A-D)

Material referido. Um ramo mandibular direito quase completo, UFRGS-PV0024K; fragmentos de angular, UFRGSPV0025K; quadrado, UFRGS-PV0026K; proótico, UFRGSPV0027K.

Descrição. UFRGS-PV0024K (Figura 5A) é um ramo mandibular quase completo, composto pelos ossos angular, gular, esplenial, dentário e pré-articular. As extremidades, anterior e posterior do ramo mandibular, porém, não estão presentes, as quais incluem parte do dentário e uma considerável porção do angular e gular. O pré-articular encontra-se fraturado anteriormente em dois locais, enquanto o osso angular é longo e com fraca ornamentação. UFRGS-PV0025K (Figura 5B) corresponde a um quadrado esquerdo fragmentado, robusto, com um processo de articulação à mandíbula proeminente. UFRGS-PV0026K (Figura 5C), por sua vez, corresponde a um osso angular esquerdo, e difere daquele presente no ramo mandibular supracitado por ser de menor tamanho e apresentar ornamentação mais proeminente em sua face medial. O espécime UFRGS-PV0027K (Figura 5D) é aqui tentativamente identificado como proótico, por sua morfologia robusta e pela presença de um forame, identificado como sendo o canal jugular, local de passagem do nervo facial.

Discussão. A diversidade de peixes do táxon Actinistia, no Mesozoico do Brasil, é representada por apenas dois gêneros: Axelrodichthys Maisey, 1986 e Mawsonia. A única espécie de Axelrodichthys conhecida, A. araripensis Maisey, 1986 ocorre no Aptiano/Albiano do Grupo Santana, bacia do Araripe, Ceará, e na bacia de Grajaú, Maranhão (Carvalho \& Maisey, 2008).

Já o gênero Mawsonia tem seus primeiros registros com a espécie M. gigas Woodward, 1907 (in Mawson \& Woodward, 1907) no Neocomiano do Grupo Ilhas (bacia do Recôncavo, Bahia) e do Grupo Areado (bacia Sanfranciscana, Minas Gerais) (Carvalho \& Maisey, 2008); M. ubangiana Casier, 1961 no Neocomiano do Zaire, África, e Mawsonia sp. no Neocomiano de Níger, também na África (Wenz, 1981). Brito \& Reis (1999) relataram a ocorrência de Mawsonia na bacia de Iguatu. Os registros mais recentes referem M. gigas e $M$. brasiliensis para o Albiano do Grupo Santana, $M$. cf. gigas para o Albiano da bacia do Grajaú (Dutra \& Malabarba, 2001), M. gigas para o Cenomaniano da bacia de São Luís 


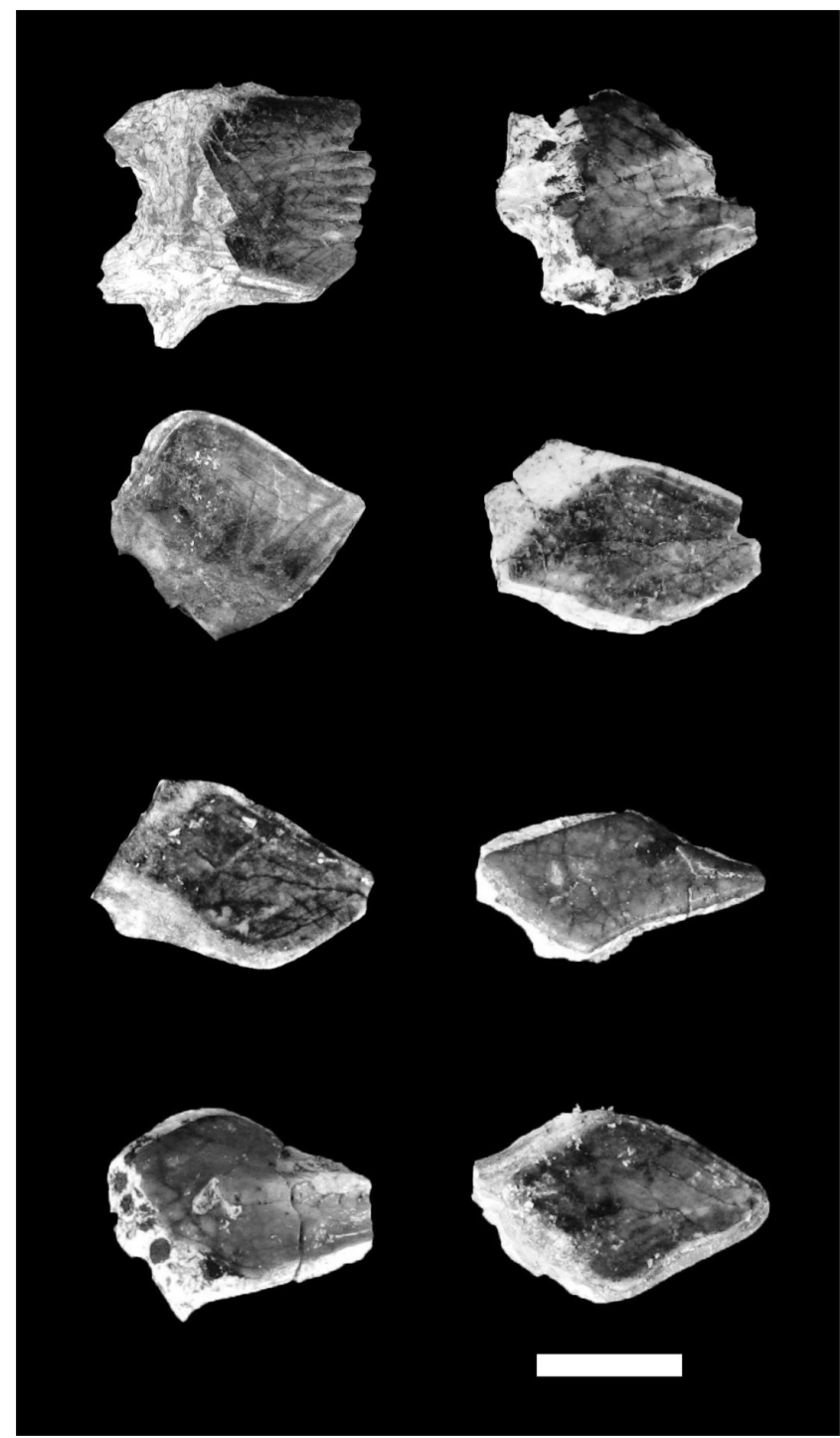

Figura 4. Escamas ganoides atribuídas a Lepidotes sp. Escala = $10 \mathrm{~mm}$.

Figure 4. Ganoid scales referred to Lepidotes sp. Scale bar $=10 \mathrm{~mm}$.

(Medeiros \& Vilas Bôas, 1999; Carvalho, 2002), Mawsonia sp. para o Cenomaniano da Laje do Coringa (Medeiros, 2001), M. lavocati Tabaste, 1963 para o Albiano do Marrocos, $M$. libyca Weiler, 1935 para o Albiano do Egito e M. tegamensis Wenz, 1973 para o Aptiano de Níger (Cloutier \& Forey, 1991; Yabumoto, 2002).

O caráter fragmentário do material da bacia de Lima Campos torna difícil sua atribuição precisa a qualquer um dos dois gêneros de peixes celacantiformes reportados para o Brasil ou oeste da África. É importante considerar a possibilidade da presença de táxons africanos nas bacias interiores do nordeste devido à íntima relação entre os continentes sulamericano e africano, durante o Eocretáceo.

A presença do canal jugular bem desenvolvido no proótico (espécime UFRGS-PV0027K) reforça a identificação como Mawsonia, pois em Axelrodichthys este forame é extremamente pequeno (Maisey, 1991). Além disso, apenas o gênero Mawsonia foi encontrado em sedimentos dulciaquícolas, enquanto Axelrodichthys ocorre em concreções do nível mais superior, marinho, do Grupo Santana. Não se descarta, entretanto, a possibilidade de uma nova espécie para a bacia de Lima Campos.

A distribuição paleobiogeográfica de Mawsonia sugere uma zona de endemismo, restrita ao nordeste e sudeste do Brasil e noroeste da África no Neocomiano. O oceano Atlântico Sul, ainda em processo de formação, não possuía ligação com o Mar de Tétis, o que restringia as migrações marinhas destes animais. Com a evolução do rifte, do sul para o norte, separando os continentes, sul-americano e africano, uma barreira biogeográfica foi eliminada. O oceano Atlântico Sul ganhou conexão com o Mar de Tétis e isto teria permitido a dispersão de Mawsonia em direção ao norte, atingindo áreas correspondentes ao atual Egito (Maisey, 1991). Os espécimes aqui descritos representam o primeiro registro do gênero Mawsonia para a bacia de Lima Campos.

\section{CROCODYLOMORPHA Walker, 1968 CROCODYLIFORMES Benton \& Clark, 1988 MESOEUCROCODYLIA Whetstone \& Whybrow, 1983 Gen. et sp. indet.} (Figura 6)

Material referido. Osteodermo completo, UFRGS-PV0020K e molde externo de osteodermo, UFRGS-PV0023K.

Descrição. UFRGS-PV0020K é um osteodermo completo pertencente a um mesoeucrocodilo de afinidade incerta. Possui forma hexagonal, com aproximadamente $35 \mathrm{~mm}$ de diâmetro. É fortemente ornamentado por depressões profundas de formato semicircular ou quadrangulares e possui as extremidades levemente arredondadas. A face inferior apresenta alguns forames, mas não há sinal de ranhuras. O espécime UFRGS-PV0023K, uma pequena porção de arenito avermelhado, preservou em uma de suas faces, o molde externo de um osteodermo. Este molde apresenta sinais de ornamentação semelhantes às de UFRGS-PV020K.

Discussão. A ornamentação predominante na forma de depressões regulares poligonais, formato hexagonal, a presença de múltiplas facetas de articulação e a inexistência de uma simetria preferencial denotam um osteodermo de posição ventral de um crocodilomorfo mesoeucrocodilo (Kalin, 1955; Steel, 1973; Benton \& Clark, 1988; Pol \& Gasparini, 2009). O tamanho do osteodermo sugere um animal de grande porte e de hábitos aquáticos. Do ponto de vista taxonômico, muito pouco se pode inferir a partir de apenas um osteodermo isolado, e quaisquer atribuições do material aqui descrito a algum dos gêneros conhecidos teriam caráter puramente especulativo sendo, assim, omitidas. Por outro lado, o UFRGS-PV0020K é incompatível em tamanho com os osteodermos de Susisuchus jaguaribensis Fortier \& Schultz, 2009, um crocodilomorfo neossúquio de pequeno porte, proveniente de outro afloramento da bacia de Lima Campos (Fortier \& Schultz, 2009).

Do ponto de vista paleoecológico, crocodilianos são indicadores de clima quente e úmido (Brochu, 2001) e a presença de um mesoeucrocodilo em sedimentos de ori- 


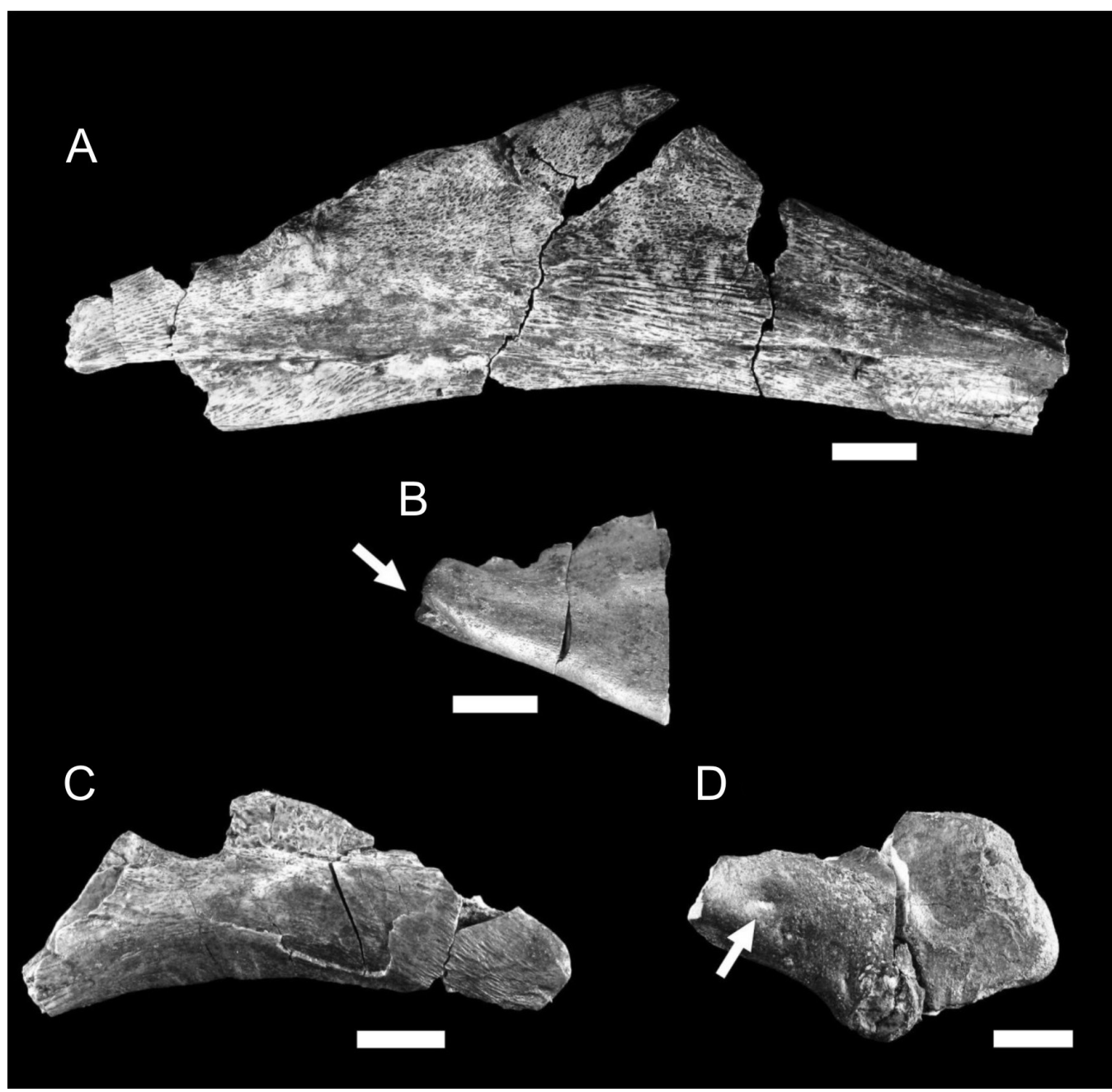

Figura 5. Elementos cranianos e mandibulares atribuídos a Mawsonia sp. A, UFRGS-PV0024K, ramo mandibular em vista medial; B, UFRGSPV0025K, fragmento de quadrado em vista lateral (a seta indica o processo de articulação à mandíbula); C, UFRGS-PV0026K, fragmento de angular em vista medial; D, UFRGS-PV0027K, fragmento de proótico em vista ventral (a seta indica o canal jugular). Escalas = $10 \mathrm{~mm}$.

Figure 5. Cranial and mandibular elements referred to Mawsonia sp. A, UFRGS-PV0024K, mandibular ramus in medial view; B, UFRGSPV0025K, fragment of quadrate in lateral view (the arrow shows the mandibular articulation process); C, UFRGS-PV0026K, fragment of angular in medial view; D, UFRGS-PV0027K, fragment of prootic in ventral view (the arrow shows the jugular channel). Scale bars = $10 \mathrm{~mm}$.

gem fluvial, associado a uma fauna de peixes, tais como os acima descritos, é coerente com a hipótese de que o conjunto destes fósseis constitui uma assembleia de organismos de água doce.

Em termos tafonômicos, a ocorrência de osteodermos isolados não indica, a princípio, a possibilidade de que sejam encontrados, no mesmo afloramento, outros restos esqueletais do mesmo indivíduo, já que osteodermos apresentam baixa densidade e são facilmente transportáveis em regimes fluviais (Holz \& Simões, 2002).

\section{CONSIDERAÇÕES FINAIS}

Os achados aqui descritos trazem importantes acréscimos ao conhecimento da fauna cretácica da bacia de Lima Campos e dos padrões de distribuição paleobiogeográfica dos táxons abordados. É importante salientar que informações mais completas e detalhadas poderão ser obtidas na medida em que forem estabelecidas coletas sistemáticas e novas pesquisas na região. Em um único afloramento da bacia de Lima Campos foram encontrados três dentes de tubarão hibodontídeo identificados como Planohybodus sp., além de grande número de escamas ganoides atribuídas a Lepidotes sp., um ramo mandibular, fragmentos de quadrado, angular e proótico de Mawsoniidae, um osteodermo e um molde externo de osteodermo de mesoeucrocodilo, demonstrando a quantidade e diversidade de fósseis existentes na região.

Dentre os táxons aqui reportados, alguns constituem registros inéditos para o Cretáceo brasileiro (como a presença do tubarão Planohybodus) e, mais localmente, para a fauna da bacia de Lima Campos (peixes Mawsoniidae). Tais registros ampliam a distribuição paleobiogeográfica destes animais, contribuindo, por exemplo, na verificação de zonas de endemismo e posterior dispersão, como no caso do gênero Mawsonia. 


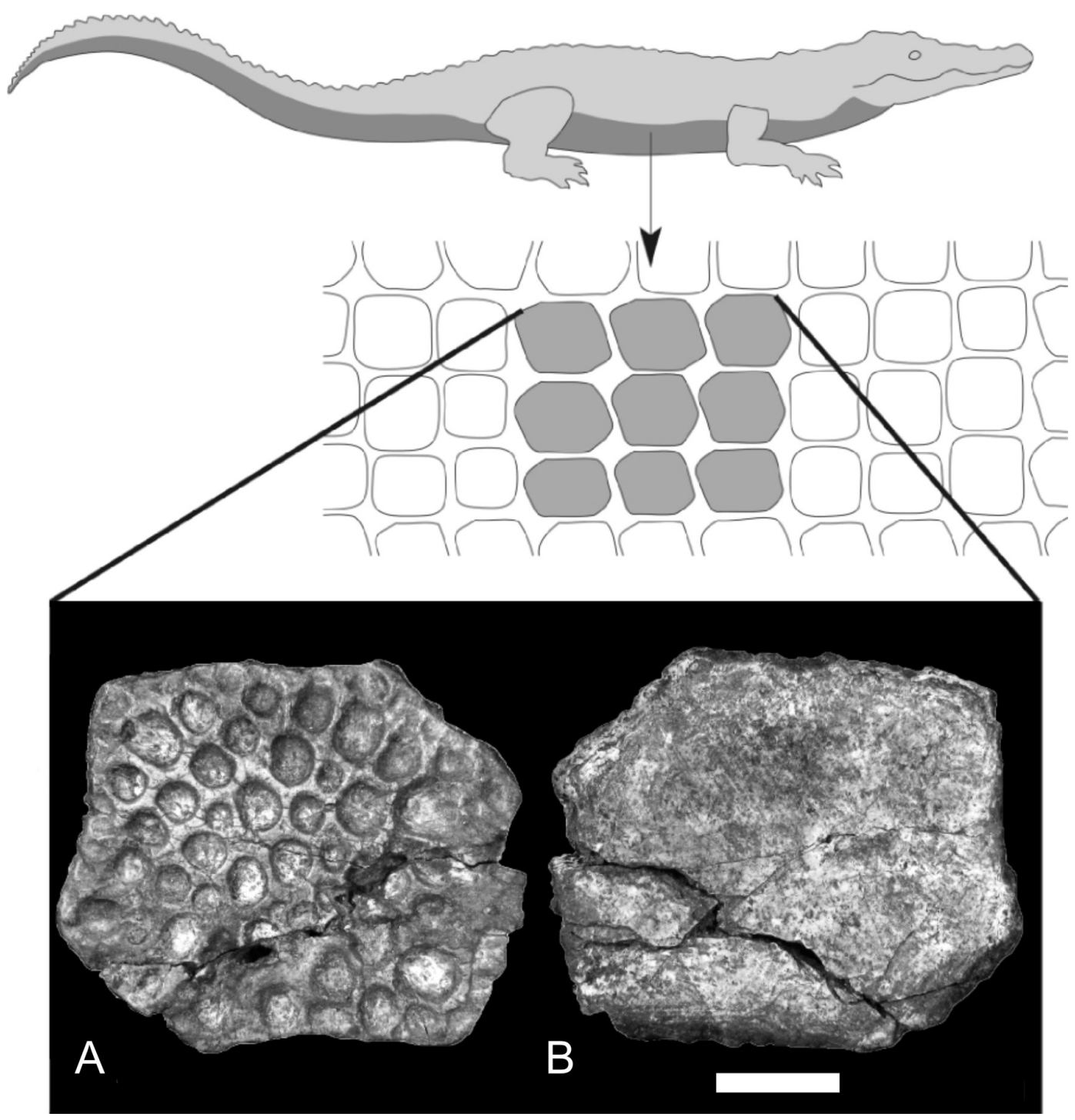

Figura 6. Osteodermo de crocodilomorfo. UFRGS-PV0020K: A, vista dorsal; B, vista ventral. Escala = $10 \mathrm{~mm}$.

Figure 6. Crocodylomorph osteoderm. UFRGS-PV0020K: A, dorsal view; B, ventral view. Scale bar = $10 \mathrm{~mm}$.

O presente trabalho revela o elevado, e ainda pouco explorado, potencial paleontológico da bacia de Lima Campos. $\mathrm{O}$ estudo de faunas associadas ao contexto de fragmentação do Gondwana é de grande valor para compreender a importância deste evento como agente modificador da paleobiota. As recentes descobertas na bacia de Lima Campos, dentre outras bacias, indicam que as pequenas bacias sedimentares interiores do nordeste brasileiro são merecedoras de um maior e sistemático esforço de pesquisa, pois apenas a paleofauna e a paleoflora da bacia do Araripe têm sido estudada com suficiente atenção.

\section{AGRADECIMENTOS}

Os autores agradecem a V. Porto e "Júnior" pela ajuda em trabalho de campo; a B. Horn e aos revisores anônimos que contribuíram significativamente na qualidade do manuscrito.
Ao Centro de Microscopia Eletrônica da UFRGS pelas fotografias em MEV. Ao Conselho Nacional de Pesquisa e Desenvolvimento Científico e Tecnológico-CNPq pelas bolsas concedidas aos três primeiros autores e pelo auxílio financeiro (projeto 78537/2008-5). À Fundação Cearense de Apoio ao Desenvolvimento Científico e Tecnológico-FUNCAP pela bolsa concedida a MSSV (projeto BPI-03341-1.07/08).

\section{REFERÊNCIAS}

Arima, N. 2007. Análise estratigráfica da bacia do Iguatu. Programa de Pós-Graduação em Geociências, Universidade Federal do Rio Grande do Sul, Porto Alegre, Dissertação de Mestrado, 149 p.

Benton, M.J. \& Clark, J. 1988. Archosaur phylogeny and the relationships of the Crocodylia. In: M.J. Benton (ed.) The Phylogeny and Classification of Tetrapods. Volume 1: Amphibians, Reptiles, Birds, Systematics Association Special Volume n 35A, p. 295-338. 
Beurlen, K. \& Mabesoone, J.M. 1969. Bacias cretáceas intracontinentais do nordeste do Brasil. Notas Geomorfológicas, 9(18):19-34.

Brito, P.M. \& Reis, M.F. 1999. Presença de Mawsonia (Actinistia; Coelacanthidae) no Cretáceo Inferior da bacia Iguatu (NE do Brasil) com comentários sobre a distribuição desses celacantídeos: In: CONGRESSO BRASILEIRO DE PALEONTOLOGIA, 16, 1999. Boletim de Resumos, Crato, SBP, p. 29-30.

Brochu, A. 2001. Crocodylian snouts in space and times: phylogenetic approaches toward adaptive radiation. American Zoologist, 41:564-585.

Campos, M.; Braga, A.P.G.; Mello, A.A.; Souza, E.M.; Silva, F.A.F \& França, J.B. 1979. Projeto Rio Jaguaribe. Brasília, Ministério das Minas e Energia, Departamento Nacional da Produção Mineral, 149 p. (Série Geologia 4).

Cappetta, H.; Duffin, C. \& Zidek, J. 1993. Chondrichthyes. In: J.M. Benton (ed.) The fossil record 2, Chapman \& Hall, p. 593-609.

Carvalho, M.S.S. 2002. O gênero Mawsonia (Sarcopterygii, Actinistia) no Cretáceo das bacias Sanfranciscana, Tucano, Araripe, Parnaíba e São Luís. Programa de Pós-Graduação em Geologia, Universidade Federal do Rio de Janeiro, Tese de Doutorado, 208 p.

Carvalho, M.S.S. \& Maisey, J. 2008. New occurence of Mawsonia (Sarcopterygii: Actinistia) from the Early Cretaceous of the Sanfranciscana Basin, Minas Gerais, southeastern Brazil. In: L. Calvin, A. Longbottom \& M. Richter (eds.) Fishes and the break-up of Pangaea, Geological Society Special Publications 295, p. 109-144.

Cavalcanti, V.M.M. \& Viana, M.S.S. 1992. Considerações sobre o Cretáceo da bacia de Iguatu, nordeste do Brasil. Anais da Academia Brasileira de Ciências, 64(1):63-70.

Cloutier, R. \& Forey, P.L. 1991. Diversity of extinct and living actinistian fishes (Sacropterygii). Environmental Biology of Fishes, 32:59-74.

Dutra, M.F.A. \& Malabarba, M.C.S.L. 2001. Peixes do AlbianoCenomaniano do Grupo Itapecuru no estado do Maranhão, Brasil. In: D.F. Rossetti; A.M. Góes \& W. Truckenbrodt (eds.) O Cretáceo na bacia de São Luís-Grajaú, Museu Paraense Emílio Goeldi, p. 191-208.

Figueiredo, A.E.Q. 2009. Estudo tafonômico de afloramentos contendo vertebrados fósseis das bacias do Iguatu, Cretáceo Inferior, Nordeste do Brasil. Programa de Pós-Graduação em Geociências, Universidade Federal do Rio Grande do Sul, Dissertação de Mestrado, $111 \mathrm{p}$.

Figueiredo, A.E.Q.; Fortier, D.C. \& Schultz, C.L. 2008. Indicação da presença de crododilomorfos aquáticos na bacia de Lima Campos através de um osteodermo: In: CONGRESSO LATINOAMERICANO DE PALEONTOLOGIA DE VERTEBRADOS, 3, 2008. Libro de Resúmenes, Neuquén, p. 94.

Fortier, D.C. 2008. Novos registros fósseis das bacias do Iguatu, Eocretáceo, estado do Ceará. Programa de Pós-Graduação em Geociências, Universidade Federal do Rio Grande do Sul, Dissertação de Mestrado, 116 p.

Fortier, D.C. \& Schultz, C.L. 2006. Novos registros fósseis das bacias do Iguatu, Centro-oeste do estado do Ceará. In: SEMANA ACADÊMICA DOS ALUNOS DE PÓS-GRADUAÇÃO EM GEOCIÊNCIAS, 1, 2006. Livro de Resumos, Porto Alegre, UFRGS, p. 51-54.

Fortier, D.C. \& Schultz, C.L. 2009. A new neosuchian crocodylomorph (Crocodyliformes, Mesoeucrocodylia) from the Early Cretaceous of north-east Brazil. Palaeontology, 52(5):991-1007. doi:10.1111/j.1475-4983.2009.00894.x

Gallo, V. \& Brito, P.M. 2004. An overview of Brazilian semionotids, In: G. Arratia \& A. Tintori (eds.) Mesozoic Fishes 3. Systematics, paleoenvironments and biodiversity, Verlag Dr. Friedrich Pfeil, p. 253-264.

Holz, M. \& Simões, M.G. 2002. Elementos Fundamentais de Tafonomia. $1^{\mathrm{a}}$ ed. Porto Alegre, Editora da Universidade/ UFRGS, $231 \mathrm{p}$.

Kalin, J. 1955. Crocodilia. In: J. Piveteau (ed.) Traité de Paléontologie 5, Masson \& Cie Editeurs, p. 695-784.

Leonardi, G. \& Spezzamonte, M. 1994. New tracksites (Dinosauria: Theropoda and Ornithopoda) from the Lower Cretaceous of the Ceará, Brazil. Studi Trentini di Scienze Naturali, Acta Geologica, 69:61-70.

Mabesoone, J.M. \& Campanha, V.A. 1974. Caracterização estratigráfica dos grupos Rio do Peixe e Iguatu. Estudos Sedimentológicos, 3/4:22-41.

Maisey, J.G. 1991. Santana fossils: an illustrated Atlas. $1^{\mathrm{a}} \mathrm{ed}$ Neptune City, T.F.H. Publishers, 459 p.

Medeiros, M.A. 2001. A Laje do Coringa (Ilha do Cajual, bacia de São Luís, Baía de São Marcos, MA): conteúdo fossilífero, bioestratonomia, diagênese e implicações na paleobiogeografia do Mesocretáceo do nordeste brasileiro. Programa de Pós-Graduação em Geociências, Universidade Federal do Rio Grande do Sul, Tese de Doutorado, 137 p.

Medeiros, M.A. \& Vilas Bôas, I. 1999. Ocorrência de uma paleocomunidade continental do Cenomaniano (Cretáceo Superior) do Nordeste do Brasil: In: JORNADAS ARGENTINAS DE PALEONTOLOGIA DE VERTEBRADOS, 15, 1999. Programas y Resúmenes, APA, La Plata, p. 18.

Moraes, L.J. 1924. Serras e montanhas do Nordeste. Rio de Janeiro, Ministério de Viação e Obras Públicas, Inspetoria de Obras contra as Secas, Geologia, (Série 1D, Publicação 58).

Mussa, D.; Babinski, M.E.C.B.O.; Dino, R. \& Arai, M. 1991. A presença do gênero Pseudofrenelopsis Nathorst na bacia mesozóica de Lima Campos, estado do Ceará, Brasil: In : CONGRESSO BRASILEIRO DE PALEONTOLOGIA, 12, 1991. Boletim de Resumos, SBP, São Paulo, p. 115.

Pol, D. \& Gasparini, Z. 2009. Skull anatomy of Dakosaurus andiniensis (Thalattosuchia: Crocodylomorpha) and the phylogenetic position of Thalattosuchia. Journal of Systematic Paleontology, 7(2):163-197. doi: 10.1017/S1477201908002605

Rees, J. 2008. Interrelationships of Mesozoic hybodont sharks as indicated by dental morphology: preliminary results. Acta Geologica Polonica, 58(2):217-221.

Rees, J. \& Underwood, C.J. 2008. Hybodont sharks from the English Bathonian and Callovian (Middle Jurassic). Palaeontology, 51:117-147. doi:10.1111/j.1475-4983.2007.00737.x

Small, H.L. 1913. Geologia e suprimento d' água subterrânea no Ceará e parte do Piauhy. Rio de Janeiro, Ministério da Viação e Obras Públicas, Inspetoria Federal de Obras contra as Secas, 81 p. (Série 7D, Publicação 25).

Steel, R. 1973. Crocodylia. In: O. Kuhn (ed.) Handbuch der Paläoherpetologie, Gustav Fischer, 116 p.

Tinoco, I.M. \& Mabesoone, J.M. 1975. Observações paleocológicas sobre as bacias mesozóicas de Iguatu, Rio do Peixe e intermediárias: In: SIMPÓSIO DE GEOLOGIA DO NORDESTE, 7, 1975. Atas, Fortaleza, p. 95-107.

Vogel, M.A.C. 1976a. Contribuição à paleontologia da bacia de Iguatu: In: CONGRESSO BRASILEIRO DE GEOLOGIA, 29, 1976. Resumos, Ouro Preto, SBG, p. 384. 
Vogel, M.A.C. 1976b. Sobre um semionotídeo da bacia de Iguatu, Ceará. Programa de Pós-graduação em Geologia, Universidade Federal do Rio de Janeiro, Dissertação de Mestrado, 63 p.

Yabumoto, Y. 2002. A new coelacanth from the Early Cretaceous of
Brazil (Sarcopterygii, Actinistia). Paleontological Research, 6(4):343-350.

Received in May, 2010; accepted in March, 2011. 